\title{
Distribuição espacial de espécies vegetais no topsoil: implicações na recuperação de áreas mineradas
}

\author{
Gleisson de Oliveira NASCIMENTO ${ }^{1 *}$, José Aldo Alves PEREIRA ${ }^{2}$, Dalmo Arantes BARROS ${ }^{3}$, \\ Rossi Allan SILVA², Josimar Batista FERREIRA4 ${ }^{4}$, Railma Pereira MORAES ${ }^{2}$, \\ Luís Antônio Coimbra BORGES ${ }^{2}$
}

\author{
${ }^{1}$ Programa de Pós-Graduação em Ciências Ambientais, Universidade Federal do Acre, Campus Floresta, Cruzeiro do Sul, AC, Brasil. \\ ${ }^{2}$ Programa de Pós-Graduação em Engenharia Florestal, Universidade Federal de Lavras, Lavras, MG, Brasil. \\ ${ }^{3}$ Universidade do Estado de Minas Gerais, Passos, MG, Brasil. \\ ${ }^{4}$ Centro de Ciências Biológicas e da Natureza, Universidade Federal do Acre, Rio Branco, AC, Brasil. \\ *E-mail: gleisson_czsac@yahoo.com.br
}

Recebido em julho/2018; Aceito em junho/2019.

\begin{abstract}
RESUMO: A distribuição espacial das espécies vegetais no topsoil antes da mineração é ferramenta importante e pode fundamentar atividades de recuperação de áreas mineradas. Neste trabalho foi realizada uma análise da distribuição espacial de quatro espécies com abordagem sobre a dinâmica da regeneração em ambientes campestres. Amostras da camada superficial do solo (cinco cm) foram coletadas em agosto de 2011 e março de 2012 em áreas de campos de altitude na região do Planalto de Poços de Caldas, Minas Gerais. As amostras foram dispostas em bandejas e a germinação avaliada em casa de vegetação. Quatro espécies com maior representatividade de germinação foram selecionadas para verificar os padrões de distribuição nos períodos seco e chuvoso por meio da análise de krigagem, e destacou-se a distribuição de $A$. fastigiatum sugerindo sua potencial indicação para recuperação de áreas após mineração.
\end{abstract}

Palavras-chave: krigagem; áreas degradadas; mapeamento vegetal.

\section{Space distribution of species in topsoil: implications to recovery of mining areas}

\begin{abstract}
The spatial distribution of plant species in topsoil prior to mining is an important tool and may support recovery activities for mined areas. In this work an analysis of the spatial distribution of four species was carried out to verify its distribution with approach on the dynamics of regeneration in rural environments. Samples of the topsoil $(5 \mathrm{~cm})$ were collected in August 2011 and March 2012 in areas of altitude fields in the Planalto region of Poços de Caldas, Minas Gerais. They were arranged in trays and evaluated in a greenhouse. Four species with greater germination representativeness were selected to verify distribution patterns in dry and rainy periods through kriging analysis. Therefore, differences in the spatial distribution of the species were identified, highlighting the distribution of $A$. fastigiatum suggesting its potential indication for recovery of areas after mining.
\end{abstract}

Keywords: kriging; degraded areas; vegetation mapping.

\section{INTRODUÇÃO}

A diversidade da vegetação em ambientes naturais é produto de uma história de eventos de recrutamento, associados a distúrbios, tais como incêndios, vento, doenças, predação de animais (RENISON et al., 2011), e que são influenciados inclusive por mudanças do uso da terra (PUNO et al., 2019).

Estudos sobre a distribuição da vegetação são determinantes para identificar padrões de colonização das espécies em um determinado ambiente natural. E no caso específico da mineração, o conhecimento da dinâmica da composição de espécies vegetais pode auxiliar na recuperação da área após as atividades de extração do minério.

Em atividades de recuperação de áreas de mineração de bauxita, a camada superficial do solo (topsoil) é estocada, e após a lavra é retornada, servindo como fonte de regeneração do ambiente (BARROS et al., 2012). Assim, o principal objetivo é restabelecer uma paisagem estável e desenvolver o uso adequado da terra após a exploração do minério (GUZMAN et al., 2019).
A distribuição das espécies no banco de sementes do solo e a forma de conduzir a recuperação da área são importantes para o sucesso das atividades, pois podem influenciar na dinâmica da distribuição das espécies ao longo do tempo de estocagem do topsoil (MORAES et al., 2017). Nesse sentido, o conhecimento do comportamento dos propágulos do solo é essencial para buscar estratégias de recuperação em ambientes montanos (SHANG et al., 2016).

A análise da distribuição dos propágulos das principais espécies presentes no topsoil em campos de altitudes sobre corpos de bauxita, antes da atividade de mineração, é uma ferramenta importante de gestão, que auxilia na tomada de decisão quanto às atividades a serem aplicadas durante a recuperação ambiental (MEIRA JUNIOR et al., 2017). Ao expressar os efeitos de diferentes distúrbios sobre mudanças na distribuição espacial das espécies, pode-se sugerir o aperfeiçoamento de técnicas no intuito de amenizar potenciais ameaças à biodiversidade (LEMENIH; TEKETAY, 2006).

$\mathrm{Na}$ geoestatística, por exemplo, vem-se aprimorando técnicas que possibilitam inferir a variabilidade espacial de 
parâmetros de interesses que permitem a elaboração de mapas temáticos, sendo a krigagem ordinária uma técnica frequentemente usada como método de interpolação de dados para áreas não amostradas (PEREIRA et al., 2013). A principal questão no presente trabalho foi entender a dinâmica da distribuição espacial de quatro espécies vegetais que ocorrem em campos altimontanos. Assim, o presente trabalho foi proposto com o objetivo de realizar uma análise da distribuição espacial de espécies vegetais que compartilham os períodos seco e chuvoso no topsoil sobre corpos de bauxita em campos de altitude na região do Planalto de Poços de Caldas - MG utilizando o método da krigagem ordinária, no intuito de aferir uma projeção da dinâmica de recomposição vegetal após alteração da área sob a interferência da mineração.

\section{MATERIAL E MÉTODOS}

2.1. Descrição da área de estudo e coleta de dados

A área de estudo corresponde a região do Planalto de Poços de Caldas (Figura 1), localizada na divisa entre os estados de Minas Gerais e São Paulo.

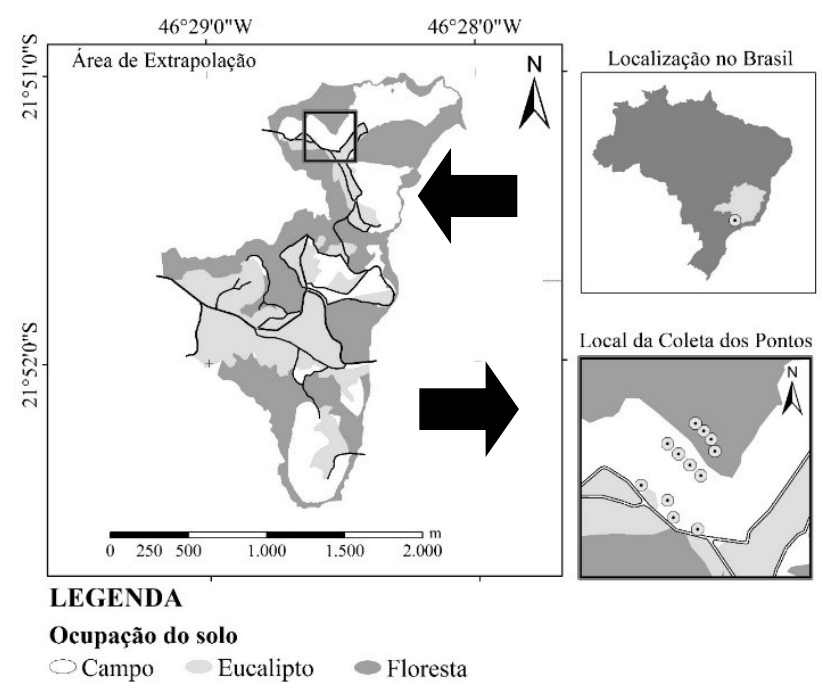

Figura 1. Localização da área de coleta e de extrapolação dos dados. Planalto de Poços de Caldas - Minas Gerais, Brasil.

Figure 1. Location of the data collection and extrapolation area. Planalto de Poços de Caldas - Minas Gerais, Brazil.

O Planalto de Poços de Caldas abriga formações florestais classificadas como Floresta Estacional Semidecidual AltoMontana (MEIRELES et al., 2008), havendo a ocorrência de fitosionomias de campos de altitude. O clima mesotérmico é do tipo Cwb, segundo classificação de Köppen atualizada por Alvares et al. (2014).

A coleta do material propagativo (topsoil) foi realizada através de amostragens do solo, na profundidade de $5 \mathrm{~cm}$ a partir da camada superficial, considerando-se esta camada do solo como a de maior concentração de propágulos das espécies regenerantes (MULLER et al., 2014).

A área da coleta (aproximadamente 2 ha) foi dividida em 3 subáreas, onde foram lançadas 12 amostras compostas (4 amostras/subárea), sendo que cada amostra foi formada por 6 subamostras (NASCIMENTO et al., 2017). A divisão em subáreas ocorreu como forma de minimizar a interferência da vegetação do entorno. Neste estudo foram realizadas duas amostragens, uma em agosto de 2011 (período seco), e outra em março de 2012 (período chuvoso), com intuito de identificar diferenças na quantidade e distribuição das espécies nos dois períodos, de acordo com a sazonalidade local. As coordenadas geográficas dos pontos centrais das amostras foram coletadas e as áreas de coleta foram demarcadas com piquetes.

As amostras do topsoil coletadas foram conduzidas ao viveiro florestal da Universidade Federal de Lavras - UFLA, espalhadas sobre $1,5 \mathrm{~cm}$ de leito de areia esterilizada em autoclave, e em seguida foram dispostas em bandejas plásticas.

$\mathrm{O}$ método utilizado para quantificação dos indivíduos presentes no conteúdo do topsoil foi o da quantificação da emergência de plântulas (NASCIMENTO et al., 2017). Após atingirem uma altura aproximada de $3 \mathrm{~cm}$, as plântulas foram transplantadas para vasos de $5 \mathrm{~kg}$, contendo substrato coletado na área de estudo e foram acondicionadas em casa de sombra - sombrite a 50\% de luminosidade. As espécies foram identificadas e as quatro de maior ocorrência nos períodos seco e chuvoso foram selecionadas para análise da distribuição espacial, utilizando o método da krigagem ordinária.

\subsection{Descrição do método de análise experimental}

Os dados do experimento foram organizados a partir de um delineamento inteiramente casualizado (DIC). O período de coleta das amostras (chuvoso e seco) e as espécies vegetais (selecionadas como de maior ocorrência no topsoil) foram os fatores considerados. A variável resposta avaliada foi o número de indivíduos germinados de cada uma das espécies selecionadas presentes nas amostras coletadas. Foram consideradas quatro repetições para cada área, buscando com isso controlar a possível variabilidade espacial dos indivíduos de cada espécie presentes nos cenários avaliados.

Os dados foram submetidos à análise de variância, ao nível de $95 \%$ de significância para o teste F. Nas situações as quais se encontrou significância estatística, realizou-se o teste de médias. O teste utilizado neste estudo foi o de Scott Knott, ao nível de 95\% de significância, e as análises foram realizadas no software R (R CORE TEAM, 2016).

\subsubsection{Análise Espacial}

A análise da dependência espacial foi realizada por meio da geoestatística, através do estudo variográfico. Este estudo foi constituído das etapas de geração do semivariograma experimental, ajustes de modelos teóricos ao semivariograma e seleção do melhor modelo para a representação dos dados. Foram avaliados diferentes cenários para a análise dos dados, considerando-se a variável número de indivíduos das quatro espécies selecionadas e os períodos seco e chuvoso.

Inicialmente foram gerados os semivariogramas experimentais para todos os cenários avaliados nesse estudo. A partir destes, foram ajustados os modelos esférico, exponencial e gaussiano para obtenção do conjunto de parâmetros a serem utilizados na análise de krigagem ordinária. Os ajustes foram feitos pelo Método dos Mínimos Quadrados Ponderados. A avaliação do desempenho de cada modelo se deu através da validação cruzada, conforme Vieira (2000).

A interpolação dos números de indivíduos das quatro espécies selecionadas foi realizada por meio da krigagem ordinária utilizando os modelos espaciais selecionados para cada cenário avaliado nesse estudo. Foram gerados mapas que demonstram o comportamento de dependência espacial para os números de indivíduos das quatro espécies vegetais de ocorrência em campo de altitude selecionadas nos períodos seco e chuvoso. O programa utilizado para análise 
geoestatística foi o $\mathrm{R}$, por meio do pacote geoR (R CORE TEAM, 2016).

\section{RESULTADOS}

Foram identificadas sete famílias botânicas no período chuvoso, representadas por 34 espécies. Dessas, cinco foram identificadas somente pelo gênero e 14 foram classificadas em morfoespécies. Dentre as famílias identificadas, Asteraceae apresentou maior riqueza específica neste período - sete (20,59\%), seguida de Poaceae - seis $(17,65 \%)$, Rubiaceae e Meslastomataceae, ambas representadas por duas espécies cada $(5,88 \%)$. No período seco foram identificadas 14 famílias botânicas e uma riqueza específica total de 49 espécies. Dentre as espécies, nove foram identificadas somente no nível de gênero e 11 como morfoespécies. Dentre as famílias identificadas, Poaceae apresentou maior riqueza - 13 (26,53\%), seguida de Asteraceae - seis (12,24\%), Rubiaceae e Melastomataceae, ambas com quatro espécies cada $(8,16 \%)$. As principais espécies identificadas foram Ageratum fastigiatum Gardner R. M. King \& H. Rob. $($ seco $=253$ indivíduos, chuvoso $=236$ indivíduos), Achyrocline satureioides Lam. DC. $($ seco $=224$ indivíduos, chuvoso $=31$ indivíduos), Borreria latifolia Aubl. K. Schum. $($ seco $=45$ indivíduos, chuvoso $=81$ indivíduos) e Melinis minutiflora $\mathrm{P}$.
Beauv. $($ seco $=30$ indivíduos e chuvoso $=18$ indivíduos $)$, uma vez que apresentaram posição fitossociológica de destaque nos períodos seco e chuvoso.

Neste trabalho, foi possível identificar diferença significativa da interação entre período e espécie $(F=7,4263$; $\mathrm{p}=0,0002)$, indicando que o comportamento biológico da distribuição das diferentes espécies está associado com os respectivos períodos (seco ou chuvoso) de coletas do topsoil.

Quando fixadas as espécies, ambos os períodos apresentaram significância $(\mathrm{p}=0,0001)$. Neste estudo foi possível observar que a espécie $A$. fastigiatum apresentou maior ocorrência nos dois períodos (seco e chuvoso), sendo $M$. minutiflora, uma espécie exótica, aquela com menor ocorrência.

Quando fixado o período de coleta dos dados (seco e chuvoso), a única espécie que apresentou significância foi $A$. satureioides ( $\mathrm{p}=0,0001)$, ou seja, para esta espécie houve influência dos períodos (seco e chuvoso) na sua distribuição em campos altimontanos, sendo que no período seco verificouse sua maior ocorrência no topsoil. Os parâmetros observados para distribuição de indivíduos das quatro espécies consideradas neste estudo foram distintos e sofreram influência do conjunto de dados aferidos, conforme o modelo utilizado para o ajuste (Tabela 1).

Tabela 1. Parâmetros para os semivariogramas ajustados.

Table 1. Parameters for the adjusted semivariograms.

\begin{tabular}{lccccc}
\hline \multicolumn{1}{c}{ Espécie } & Período & Modelo & Efeito pepita & Patamar & Alcance \\
\hline A. satureioides & Seco & esférico & 0,00 & 91,80 & 74,90 \\
A. satureioides & chuvoso & exponencial & 1,50 & 7,12 & 22,82 \\
A. fastigiatum & Seco & gaussiano & 18,47 & 109,82 & 54,23 \\
A. fastigiatum & chuvoso & esférico & 7,42 & 149,37 & 66,04 \\
B. latifolia & Seco & esférico & 11,11 & 201,3 & 68,58 \\
B. latifolia & chuvoso & gaussiano & 0,00 & 78,35 & 73,69 \\
M. minutiflora & seco & exponencial & 3,27 & 22,98 & 17,78 \\
M. minutiflora & chuvoso & exponencial & 0,00 & 7,52 & 93,85 \\
\hline
\end{tabular}

Nas Figuras 2 e 3 encontra-se a distribuição espacial por meio do método da krigagem ordinária de 4 espécies que ocorrem em campos de altitude na região do Planalto de Poços de Caldas, Minas Gerais. De acordo com os resultados, observou-se que a espécie $A$. fastigiatum foi predominante na área estudada, considerando-se os períodos seco e chuvoso, pois apresentou alta distribuição no topsoil coletado. Já a espécie $A$. satureioides apresentou alta distribuição no período seco e baixa no chuvoso (Figura 2). Nesse sentido, os altos padrões de distribuição de $A$. fastigiatum propiciaram a esta população ampla ocorrência na área estudada.

Além disso, observou-se também que a espécie $M$. minutiflora apresentou baixa distribuição no período seco, e média no período chuvoso (Figura 3). Entretanto, B. latifolia apresentou alta distribuição no período seco e média distribuição no período chuvoso.

\section{DISCUSSÃO}

4.1 Influência dos períodos de coleta do topsoil na distribuição das espécies

Os eventos de fenologia e distribuição de uma espécie vegetal em determinado período são determinados pela sazonalidade local (MUNHOZ; FELFILI, 2007). No período seco, por exemplo, houve maior ocorrência de indivíduos da espécie $A$. satureioides, tendo em vista seu comportamento fenológico, que geralmente apresenta floração na estação chuvosa (ARRUDA et al., 2009), sendo o pico de produção de sementes no final da estação seca. Essa é uma estratégia para emergir e iniciar o estabelecimento no topsoil logo após o início das chuvas (MUNHOZ; FELFILI, 2007).

A predominância de indivíduos no banco de propágulos no período seco, verificada neste trabalho, pode ser atribuída ao modo de dispersão, predominantemente, anemocórico de plantas herbáceas (KINOSHITA et al., 2006), que investem em grande quantidade de sementes como forma de superar ambientes de difícil colonização (NASCIMENTO et al., 2017). Espécies com estratégias de dispersão por anemocoria são comuns em formações abertas e predominam no final de períodos secos o que reflete a estratégia de reprodução com emergência dos propágulos no período chuvoso (LIEBSCH; ACRA, 2007).

A distribuição espacial das espécies no banco de sementes apresentou forte dependência em função dos diferentes períodos de coletas. Nas análises espaciais dos cenários avaliados, o número de indivíduos para cada espécie considerada apresentou dependência espacial, ou seja, observou-se que existe uma função estrutural com semivariância de comportamento modelável (LEMOS FILHO et al., 2008). Os pontos localizados a distâncias maiores que os alcances práticos foram independentes, o que reforça a ideia de que a abordagem apropriada para análise desse tipo de variável é a geoestatística, uma vez que se fossem utilizados 
os estimadores da estatística clássica, além de se violar o princípio da independência entre as amostras, haveria redundância de informação para o parâmetro de regeneração natural na área.

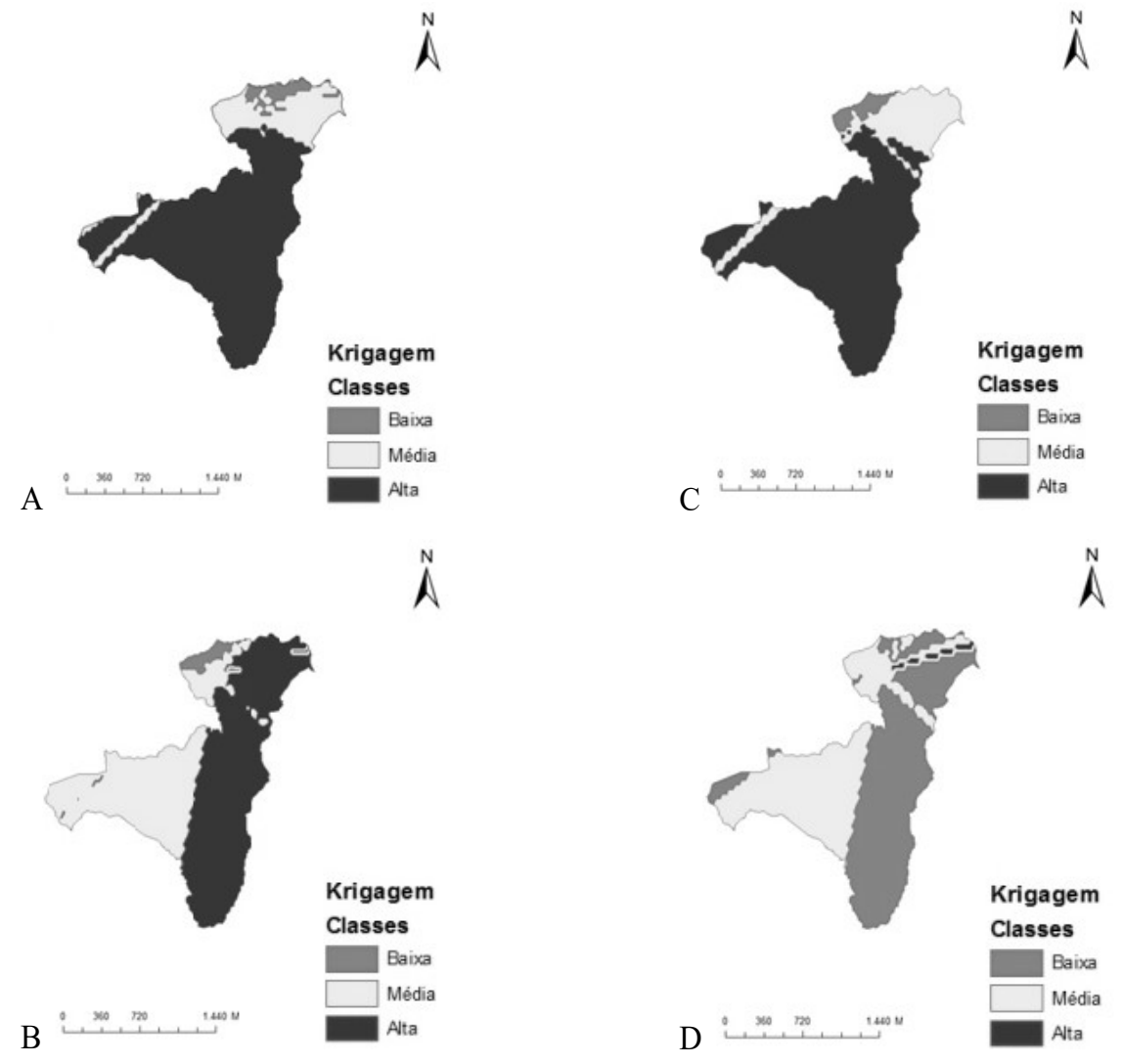

Figura 2. Distribuição para espécie $A$. fastigiatum no período seco (A) e chuvoso (B). Distribuição para espécie A. satureioides no período seco $(\mathrm{C})$ e chuvoso (D).

Figure 2. Distribution for A. fastigiatum species in dry (A) and rainy (B) season. Distribution for A. satureioides species in the dry (C) and rainy (D) season.

As estimativas realizadas com interpolação por krigagem ordinária tendem reduzir incertezas em situações com maior número de vizinhos (BELTRAME et al., 2017). Em contrapartida, baixos valores de alcance, como observados para $M$. minutiflora no período seco (Tabela 1), podem influenciar negativamente na qualidade das estimativas, uma vez que poucos pontos são usados para realização da interpolação na estimativa de valores em locais não mensurados (CORÁ et al., 2004).

Apesar de não apresentar elevada distribuição espacial na área estudada, $M$. minutiflora é uma espécie perene, altamente agressiva e compete com sucesso com a flora nativa, pois apresenta alto poder de germinação e dormência de longa duração (MARTINS et al., 2011). Isso possibilita longos períodos de viabilidade no conteúdo do topsoil, formando banco de sementes com alto potencial germinativo. Mesmo $M$. minutiflora não apresentando ameaça iminente à distribuição da flora nativa da área de estudo deste trabalho, existe uma preocupação com relação à possibilidade de expansão desta espécie, devido sua alta capacidade reprodutiva (CARMONA; MARTINS, 2010). Adicionalmente, casos de substituição de espécies invasoras sobre a população nativa levam ao aumento da competição interespecífica e diminuição da população substituída (DI et al., 2017).

Dessa forma, torna-se necessário o isolamento das áreas em processo de recuperação para evitar o estabelecimento de espécies invasoras, e também a entrada de rebanhos no local.
Portanto, é importante a elaboração de políticas ambientais para conservação e manutenção de ambientes campestres nativos como forma de otimizar as ações de recuperação dessas áreas.

4.2 Implicações da distribuição das espécies vegetais no topsoil para recuperação de áreas mineradas

O modelo de distribuição proposto para as espécies herbáceas de considerável ocorrência no topsoil sobre corpos de bauxita do Planalto de Poços Caldas sugere uma possível sucessão com predominância de $A$. fastigiatum e também a ocorrência de espécies invasoras (M. minutiflora). É certo que algumas mudanças podem ocorrer no processo de sucessão devido a possíveis alterações no banco de sementes presentes no topsoil e o estabelecimento de espécies invasoras que podem dominar a fitofisionomia (CHAPLA; CAMPOS, 2011). Apesar da reduzida escala espaço-temporal (GEORGAKARAKOS; KITSIOU, 2008), os resultados encontrados indicam uma dinâmica na distribuição diretamente relacionada com a fenologia das espécies, chamando atenção para espécies de alta distribuição espacial nos períodos seco e chuvoso, e também espécies exóticas.

No topsoil coletado em diferentes períodos foi possível identificar uma dinâmica distinta entre as espécies vegetais de ocorrência em campos de altitude, sendo que de modo geral tiveram maior ocorrência no período seco (A. fastigiatum, A. satureioides e Melinis minutiflora) quando comparado com o 
período chuvoso, no qual se identificou maior ocorrência de $B$. latifolia. Portanto, sugere-se que o estoque e armazenamento do topsoil em áreas sob a interferência da mineração de bauxita seja realizado no período seco como forma de otimizar as técnicas e contemplar uma maior dinâmica das principais espécies para fins de recuperação destas áreas após a mineração (NASCIMENTO et al., 2017).

A conservação dos ecossistemas campestres tem sido negligenciada. Levantamentos de Silveira et al. (2016) apontam que aproximadamente $15 \%$ da diversidade de plantas do Brasil está em área campestre, distribuída em $0,78 \%$ da superfície brasileira. Os campos de altitude remanescentes, geralmente, sofrem com medidas inadequadas de manejo, como a queima, a introdução e/ou facilitação de estabelecimento de espécies exóticas (MOCOCHINSKI; SCHEER, 2008). Portanto, faz-se necessária a realização de levantamentos estruturais e florísticos, bem como a análise da distribuição das espécies no banco de sementes em diferentes períodos visando a manutenção e capacidade desses ambientes em sustentar a vida e a diversidade encontrada antes de qualquer alteração ambiental.
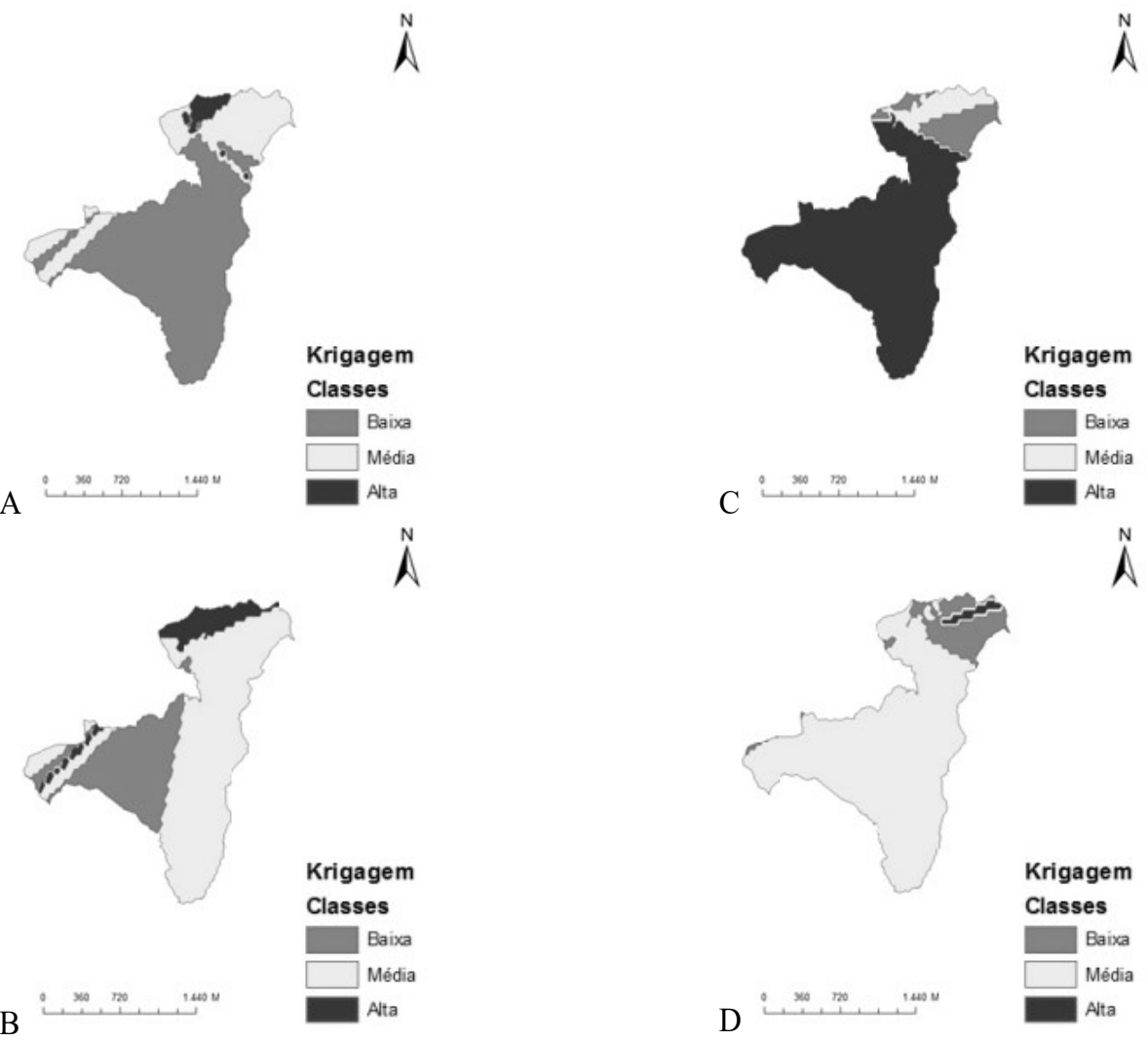

Figura 3. Distribuição para espécie M. minutiflora no período seco (A) e chuvoso (B). Distribuição para espécie B. latifolia no período seco (C) e chuvoso (D).

Figure 3. Distribution for M. minutiflora species in the dry (A) and rainy (B) season. Distribution for B. latifolia species in the dry (C) and rainy (D) season.

\section{CONCLUSÕES}

O comportamento das espécies foi distinto com relação à distribuição espacial nos diferentes períodos (seco e chuvoso). Assim, chamamos atenção para elevada distribuição espacial da espécie $A$. fastigiatum nos dois períodos, e também, para ocorrência em uma amplitude de classes considerada de baixa a média distribuição da espécie exótica $M$. minutiflora. A grande ameaça para conservação de ambientes campestres é a possibilidade das espécies exóticas dominar o ambiente e dificultar a regeneração de outras espécies, ocasionando mudanças na recomposição das áreas de vegetação campestre após atividades de mineração.

De modo geral, no período seco foi identificada maior ocorrência de A. fastigiatum e A. satureioides. Vale destacar, a elevada distribuição de $A$. fastigiatum em ambos os períodos de coleta do topsoil em áreas de campos de altitude. Assim, o estoque e armazenamento do topsoil em áreas sob a interferência da mineração de bauxita devem ser realizados no período seco.

\section{AGRADECIMENTOS}

À Coordenação de Aperfeiçoamento de Pessoal de Nível Superior (CAPES) pela concessão da bolsa de estudo. À Universidade Federal do Acre e Universidade Federal de Lavras, pelo apoio logístico e aos professores do Departamento de Ciências Florestais da Universidade Federal de Lavras - UFLA pela contribuição científica. À Companhia Brasileira de Alumínio pelo apoio para o desenvolvimento desse trabalho.

\section{REFERÊNCIAS}

ALVARES, C. A.; STAPE, J. L.; SENTElHAS, P. C.; GONÇALVES, J. L. de M.; SPAROVEK, G. Köppen's climate classification map for brazil. Meteorologische Zeitschrift, Berlin, v. 22, n. 6, p. 711-728, 2014. DOI: https://dx.doi.org/10.1127/0941-2948/2013/0507

ARRUDA, R.; FLORENCIO, C.; FIGUEIREDO, R. A. de; LIMA, M. I. S.; SALVADOR, N. N. B. Composição e fenologia de espécies herbáceas nativas em 
reflorestamento heterogêneo. Floresta, Curitiba, v. 39, n. 3, p. 525-533, 2009. DOI: http://dx.doi.org/10.5380/rf.v39i3.15352a

BARROS, D. A. de; GUIMARÃES, J. C. C.; PEREIRA, J. A. A.; BORGES, L. A. C.; SILVA, R. A.; PEREIRA, A. A. S. Characterization of the bauxite mining of the Poços de Caldas alkaline massif and its socio-environmental impacts. REM: R. Esc. Minas, Ouro Preto, v. 65, n. 1, p. 127-133, 2012. DOI: http://dx.doi.org/10.1590/S037044672012000100018

BELTRAME, R. A.; LOPES, J. C.; LIMA, J. S. de S.; QUINTO, V. M. Spatial distribuition of physiological quality of Joannesia princeps Vell. seeds. Revista Árvore, Viçosa, v. 41, n. 4, 2017. DOI http://dx.doi.org/10.1590/1806-90882017000400010

CARMONA, R.; MARTINS, C. R. Qualidade física, viabilidade e dormência de sementes recém-colhidas de capim-gordura (Melinis minutiflora P. Beauv.). Revista Brasileira de Sementes, Londrina, v. 32, n. 1, p. 077-082, 2010. DOI: http://dx.doi.org/10.1590/S010131222010000100009

CHAPLA, T. E.; CAMPOS, J. B. Soil seed bank during succession at an abandoned pasture in the upper Paraná river-floodplain, Brazil. Acta Scientiarum Biological Sciences, v. 33, n. 1, p. 59-69, 2011.

CORÁ, J. E.; ARAUJO, A. V.; PEREIRA, G. T.; BERALDO, J. M. G. Variabilidade espacial de atributos do solo para adoção do sistema de agricultura de precisão na cultura de cana-de-açúcar. Revista Brasileira Ciência do Solo, Viçosa, v. 28, n. 6, p. 1013-1021, 2004. DOI: http://dx.doi.org/10.1590/S0100-06832004000600010

DI, K.; JIAN, D.; XIAOWEI, Q.; FEI, H.; SHUJUAN, G.; XINHUI, H.; GAIHE, Y. Effect of competition on spatial patterns of oak forests on the Chinese Loess Plateau. Journal of Arid Land, v. 9, n. 1, p. 122-131, 2017. DOI: https://dx.doi.org/10.1007/s40333-016-0024-9

GEORGAKARAKOS, S.; KITSIOU, D. Mapping abundance distribution of small pelagic species applying hydroacoustics and Co-Kriging techniques. Essential Fish Habitat Mapping in the Mediterranean, v. 612, p. 155169, 2008. DOI: https://dx.doi.org/10.1007/978-1-40209141-4 12

GUZMAN, J. G.; USSIRI, D. A. N.; LAL, R. Soil physical properties following conversion of a reclaimed minesoil to bioenergy crop production. Catena, Amsterdam, v. 176, p. 289-295, 2019.

DOI: https://dx.doi.org/10.1016/j.catena.2019.01.020

KINOSHITA, L. S.; TORRES, R. B.; FORNI-MARTINS, E. R.; SPINELLI, T.; AHN, Y. J.; CONSTÂNCIO, S. S. Composição florística e síndrome de polinização e de dispersão da mata do sítio São Francisco, Campinas, SP, Brasil. Acta Botanica Brasilica, São Paulo, v. 20, n. 2, p. 313-327, 2006. DOI: http://dx.doi.org/10.1590/S010233062006000200007

LEMENIH, M.; TEKETAY, D. Changes in soil seed bank composition and density following deforestation and subsequent cultivation of a tropical dry Afromontane forest in Ethiopia. Tropical Ecology, Varanasi, v. 47, n. 1, p. 1-12, 2006.

LEMOS FILHO, L. C. de A.; OLIVEIRA, E. L. de; FARIA, M. A. de; ANDRADE, L. A. de B. Variação espacial da densidade do solo e matéria orgânica em área cultivada com cana-de-açúcar (Saccharum officinarum L.). Revista
Ciência Agronômica, Fortaleza, v. 39, n. 2, p. 193-202, 2008.

LIEBSCH, D.; ACRA, L. A. Síndromes de dispersão de diásporos de um fragmento de floresta ombrófila mista em Tijucas do Sul, PR. Revista Acadêmica, v. 5, n. 2, p. 167 175 , 2007.

DOI: http://dx.doi.org/10.7213/cienciaanimal.v5i2.9750

MARTINS, C. R.; VALL HAY, J. D.; WALTER, B. M. T.; PROENÇA, C. E. B.; VIVALDI, L. J. Impacto da invasão e do manejo do capim-gordura (Melinis minutiflora) sobre a riqueza e biomassa da flora nativa do Cerrado sentido restrito. Revista Brasil. Bot., São Paulo, v. 34, n. 1, p. 7390. 2011. DOI: http://dx.doi.org/10.1590/S010084042011000100008

MEIRA JUNIOR, M. S.; MACHADO, E. L. M.; PEREIRA, I. M.; MOTA, S. da L. L. Distribuição Espacial de Eremanthus incanus (Less). ( Asteraceae ) em duas áreas com diferentes níveis de conservação. Revista Brasileira de Biociências, Porto Alegre, v. 15, n. 1, p. 27-31, 2017.

MEIRELES, L. D.; SHEPHERD, G. J.; KINOSHITA, L. S. Variações na composição florística e na estrutura fitossociológica de uma floresta ombrófila densa altomontana na Serra da Mantiqueira, Monte Verde, MG. Revista Brasileira de Botânica, São Paulo, v. 31, n. 4, p. 559-574, 2008. DOI: http://dx.doi.org/10.1590/S010084042008000400003

MOCOCHINSKI, A. Y.; SCHEER, M. B. Campos de altitude na serra do mar paranaense: aspectos florísticos. Floresta, Curitiba, v. 38, n. 4, p. 625-640, 2008. DOI: http://dx.doi.org/10.5380/rf.v38i4.13158

MORAES, R. P.; CARVALHO, W. A. C.; PEREIRA, J. A. A.; NASCIMENTO, G. O.; BARROS, D. A. Effect of topsoil stockpiling on the viability of seed bank in field phytophysiognomies Campos de Altitude. Cerne, Lavras, v. 23, n. 3, p. 339-347, 2017. DOI: http://dx.doi.org/10.1590/01047760201723032340

MULLER, I.; MESLÉARD, F.; BUISSON, E. Effect of topsoil removal and plant material transfer on vegetation development in created Mediterranean meso-xeric grasslands. Applied Vegetation Science, v. 17, p. 246261, 2014. DOI: https://dx.doi.org/10.1111/avsc. 12059

MUNHOZ, C. B. R.; FELFILI, J. M. Reproductive phenology of an herbaceous-subshrub layer of a Savannah (campo sujo) in the Cerrado Biosphere Reserve I, Brazil. Braz. J. Biol., São Carlos, v. 67, n. 2, p. 299-307, 2007. DOI: http://dx.doi.org/10.1590/S1519-69842007000200015

NASCIMENTO, G. O.; PEREIRA, J. A. A.; BARROS, D. A.; VIANA, P. L.; CARVALHO, W. A. C.; GARCIA, P. O.; FERREIRA, J. B. Espécies vegetais potenciais para recuperação de áreas sob a influência da mineração de bauxita em campos de altitude na região de Poços de Caldas - MG. Ciência Florestal, Santa Maria, v. 27, n. 1, p. $\quad 85-96 . \quad 2017 . \quad$ DOI: http://dx.doi.org/10.5902/1980509826449

PEREIRA, G. T.; SOUZA, Z. M. de; TEIXEIRA, D. de B.; MONTANARI, R.; MARQUES JÚNIOR, J. Optimization of the sampling scheme for maps of physical and chemical properties estimated by kriging. Revista Brasileira de Ciência do Solo, Viçosa, v. 37, n. 5, p. 1128-1135, p. 2013. DOI: http://dx.doi.org/10.1590/S010006832013000500002

PUNO, R. C. C.; PUNO, G. R.; TALISAY, B. A. M. Hydrologic responses of watershed assessment to land 
cover and climate change using soil and water assessment tool model. Global Journal of Environmental Science and Management, v. 5, n. 1, p. 71-82, 2019. DOI: https://dx.doi.org/10.22034/gjesm.2019.01.06

$\mathrm{R}$ CORE TEAM. R: A language and environment for statistical computing. Vienna, Austria: R Foundation for Statistical Computing, 2016.

RENISON, D.; HENSEN, I.; SUAREZ, R. Landscape structural complexity of high-mountain Polylepis australis forests: A new aspect of restoration goals. Restoration Ecology, v. 19, n. 3, p. 390-398, 2011. DOI: https://doi.org/10.1111/j.1526-100X.2009.00555.x

SHANG, Z.; YANG, S.; WANG, Y.; SHI, J.; DING, L.; LONG, R. Soil Seed Bank and Its Relation with aboveGround Vegetation along the Degraded Gradients of Alpine Meadow. Ecological Engineering, Oxford, v. 90, p. 268-77, 2016. DOI: https://dx.doi.org/10.1016/j.ecoleng.2016.01.067

SILVEIRA, F. A. O.; NEGREIROS, D.; BARBOSA, N. P. U.; BUISSON, E.; CARMO, F. F. do; CARSTENSEN, D. W.; CONCEIÇÃO, A. A.; CORNELISSEN, T. G.; ECHTERNACHT, T.; FERNANDES, G. W.; GARCIA, Q. S.; GUERRA, T. J.; JACOBI, C. M.; LEMOS-FILHOS, J. P. Ecology and evolution of plant diversity in the endangered campo rupestre: a neglected conservation priority. Plant and Soil (Print), The Hague, v. 403, n. 12, p. 129-152. 2016. DOI: https://dx.doi.org/10.1007/s11104-015-2637-8

VIEIRA, S. R. Geoestatística em estudos de variabilidade espacial do solo. In: NOVAIS, R. F.; ALVAREZ V., V. H.; SCHAEFFER, C. E. G. R. Tópicos em ciência do solo. Viçosa: Sociedade Brasileira de Ciência do Solo, 2000. v. 1. p. 1-54. 